\title{
Is Abnormality in the Conventional Anorectal Manometry Really Abnormal?
}

\author{
Hyun II Seo, MD, Jung Ho Park, MD, and Chong II Sohn, MD*
}

Department of Gastroenterology, Kangbuk Samsung Hospital, Sungkyunkwan University School of Medicine, Seoul, Korea

A 54-year-old woman, with chronic abdominal discomfort and difficulty in defecation, was admitted to the hospital. There were no abnormal findings on abdominal X-ray and colonic transit time remained within the normal values. The result by co- lonoscopic examination was normal. Bulk-forming laxatives were prescribed for a month, but symptomatic improvement was not found at all. Then, a defecography and the high resolution anorectal manometry (HR-ARM) of ManoScan TM system (Sierra
A

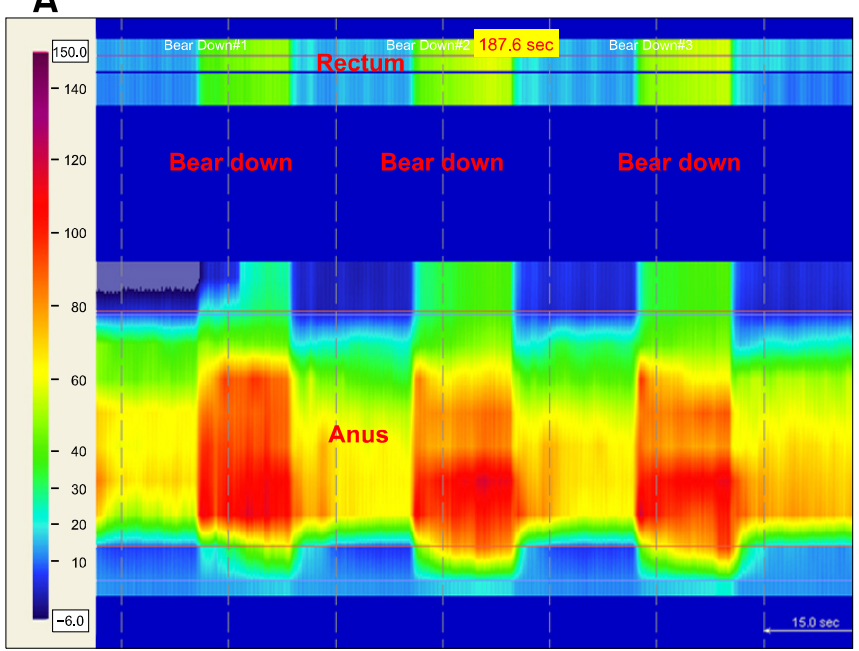

B

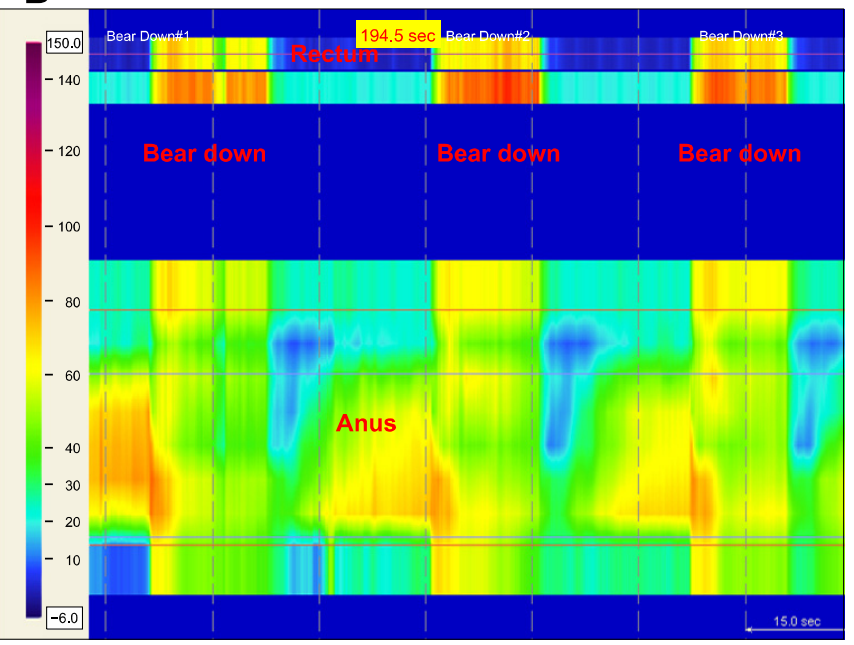

Figure 1. Colored pressure topography plots of anorectal pressure during simulated defecation in left lateral decubitus position (A) and sitting position (B). High resolution anorectal manometry (HR-ARM) finding in left lateral decubitus position revealed inappropriate increase of rectal pressure (green color) and no decrease in anal sphincter pressure (yellow to red color). This finding was consistent with pelvic floor dyssynergia (A). However, when HR-ARM was done with the patient in the sitting position, rectal pressure increased (yellow to red color) in coordination with relaxation of the anal sphincter pressure (green color), which is a normal pattern of anorectal manometry (B).

Received: March 9th, 2010 Accepted: April 6th, 2010

(c) This is an Open Access article distributed under the terms of the Creative Commons Attribution Non-Commercial License (http://creativecommons. org/licenses/by-nc/3.0) which permits unrestricted non-commercial use, distribution, and reproduction in any medium, provided the original work is properly cited.

*Correspondence: Chong II Sohn, MD

Department of Gastroenterology, Kangbuk Samsung Hospital, Sungkyunkwan University School of Medicine, 108 Pyung-dong, Jongro-gu, Seoul 110-746, Korea

Tel: +82-2-2001-2057, Fax: +82-2-2001-2610, E-mail: chongil.sohn@samsung.com

Financial support: None.

Conflicts of interest: None. 
Scientific Instruments, Los Angeles, CA, USA) were performed to differentiate functional anorectal disorders. Defecogram did not show significant findings. HR-ARM finding in left lateral decubitus position revealed the inappropriate increase of rectal pressure and no decrease in anal sphincter pressure (Fig. 1A). These findings were consistent with pelvic floor dyssynergia. However, when HR-ARM was performed with the patient in the sitting position, the increase of rectal pressure could be found in coordination with relaxation of the anal sphincter pressure (Fig. 1B). These results were consistent with the previous report obtained in the lying position; one-third showed dyssynergia and one-half could not expel artificial stool. However, when sitting with distended rectum, most patients showed a normal defecation pattern and ability to expel stool. ${ }^{1}$

During the conventional water perfusion manometry, patients are expected to be in their lateral decubitus position, which interferes with an optimal abdominal contraction and anal relaxation during simulated defecation. However, in case of HR-ARM, patients can take sitting position in commode, which is more physiological since patients can feel more comfortable and they can generate enough movement of rectum for defecation and adequate anal relaxation. ${ }^{2}$

In conclusion, defecation is best evaluated in the sitting position of patient by using HR-ARM.

\section{References}

1. Rao SS, Kavlock R, Rao S. Influence of body position and stool characteristics on defecation in humans. Am J Gastroenterol 2006; 101:2790-2996

2. Jones MP, Post J, Crowell MD. High-resolution manometry in the evaluation of anorectal disorders: a simultaneous comparison with water-perfused manometry. Am J Gastroenterol 2007;102:850-855. 